\title{
A SURVEY ON VIDEO OBJECT ABSTRACTION TECHNIQUE
}

\author{
Parul Saxena ${ }^{1} \&$ R. S. Jadon ${ }^{2}$
}

Abstract: Video object abstraction technique is a way by which a video object can be detect and track while eliminating the redundant information present in frames. It is used in numerous applications like in robot vision, monitoring the traffic, Video surveillance, Video in-painting and Simulation etc. Object abstraction is performed to confirm the existence of objects in video and to specify the location of the object, it can be anything such as humans, automobiles, birds, clouds and other moving object. In this paper we present the comparative study video abstraction technique.

Keywords - Object-based extraction, video analysis, video-object segmentation, object detection, object tracking.

\section{INTRODUCTION}

Video object tracking is difficult task because of huge amount of information is associated with video. For applying video abstraction technique video must be converted into frames. We can apply each technique in single frame. Firstly, we used the fixed camera to capture video image information, and applied the morphological erosion and dilation method to reduce the noises. Then detects objects from surveillance video using background subtraction, statistical method, temporal frame differencing and object segmentation. Here various techniques are discussed.

\section{METHODS OF VIDEO OBJECT ABSTRACTION}

In Video object abstraction numerous techniques are available. Some are frame differencing, optical flow, shape and color based representation, motion oriented representation, background subtraction, image segmentation etc. These techniques are also again used for filtering the output.

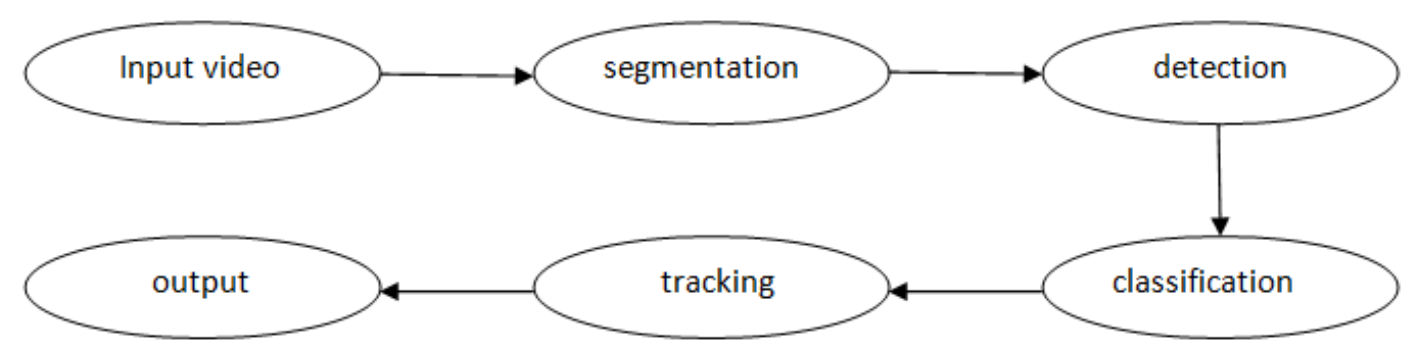

Figure 1: Steps involves in video object abstraction techniques

\subsection{Object segmentation}

The division of an image into meaningful structures is called image segmentation. And different techniques follow the image segmentation. The purpose of image segmentation is to cluster pixels of an image into image regions. We can use segmentation for image compression, object recognition, and image editing processing [1-3].

\subsubsection{Thresholding based Segmentation}

Thresholding technique is used to convert a multilevel or a grey scale image into a binary image with the help of histogram of the image.

$\mathrm{T}=\mathrm{T}[\mathrm{x}, \mathrm{y}, \mathrm{P}(\mathrm{x}, \mathrm{y}), \mathrm{f}(\mathrm{x}, \mathrm{y})]$

\footnotetext{
${ }^{1}$ MCA, MITS, Gwalior, MP, India

${ }^{2}$ MCA, MITS, Gwalior, MP, India
} 
Where $x$ and $y$ are the pixels, $P(x, y), f(x, y)$ are the points of gray level image. If $T$ depends on $f(x, y)$ then it is known as Global thresholding and if it depends upon, $\mathrm{P}(\mathrm{x}, \mathrm{y}), \mathrm{f}(\mathrm{x}, \mathrm{y})$ it is called Local thresholding and if it depends on $\mathrm{x}, \mathrm{y}, \mathrm{P}(\mathrm{x}, \mathrm{y}), \mathrm{f}(\mathrm{x}$, y) is known as Adaptive thresholding.
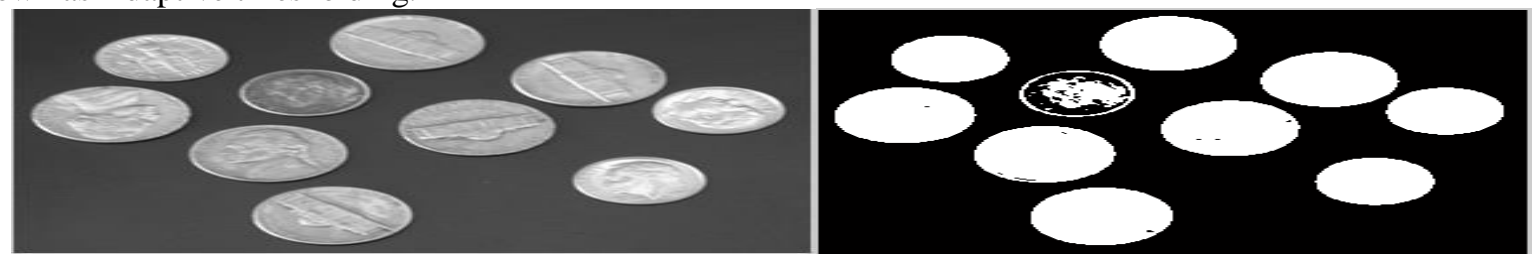

Figure 2. (a) Gray scale image (b) Resultant image after thresholding

\subsubsection{Edge Based Segmentation}

In this method the images are partitioned by identifying the edges or pixels of rapid transition in intensity. Edge Based approach is done by first detecting the edges or pixels between the different region that have change in intensity and linked those pixels to form closed boundaries.
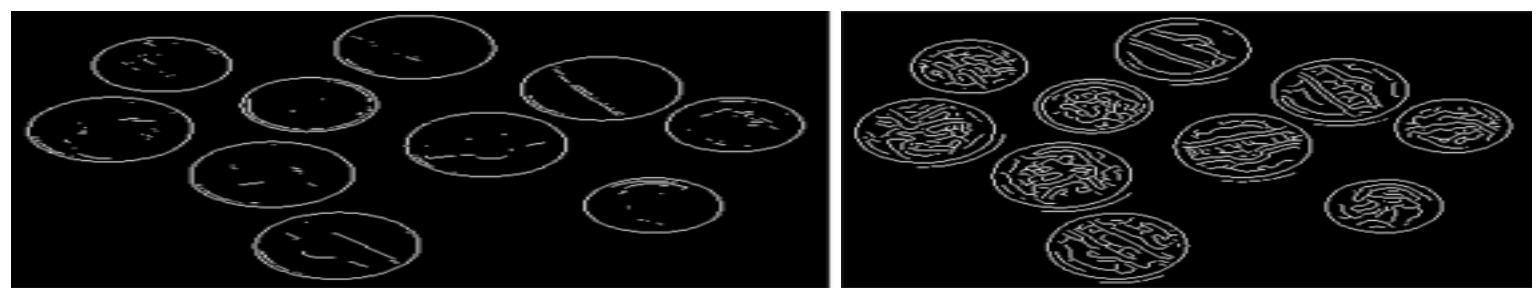

Figure 3. (a) Edges detection (b) Edges with closed connected boundaries

\subsubsection{Region Based Segmentation}

In region based segmentation technique the similar subset or pixels of an image based upon some criteria are grouped together to form image region. Image regions are constellation of connected pixels with similar belongings.

Table-1 Comparative study of segmentation techniques

\begin{tabular}{|l|l|l|l|}
\hline Segmentation Technique & Method Description & Advantages & Limitations \\
\hline Thresholding method & $\begin{array}{l}\text { Depends on the histogram } \\
\text { of an image }\end{array}$ & $\begin{array}{l}\text { There is no any } \\
\text { requirement of prior } \\
\text { knowledge of image }\end{array}$ & $\begin{array}{l}\text { It doesn't work well if too } \\
\text { many edges are present }\end{array}$ \\
\hline Edge detection approach & $\begin{array}{l}\text { Pixels having different } \\
\text { intensities }\end{array}$ & $\begin{array}{l}\text { Easily detection of the } \\
\text { edges }\end{array}$ & $\begin{array}{l}\text { This method is less } \\
\text { immune to noise }\end{array}$ \\
\hline Region based method & $\begin{array}{l}\text { Grouping of pixels having } \\
\text { similar properties and and } \\
\text { form the region }\end{array}$ & $\begin{array}{l}\text { Work well when the } \\
\text { region homogeneity } \\
\text { criteria are easily defines } \\
\text { and more immune to noise }\end{array}$ & $\begin{array}{l}\text { This technique consists of } \\
\text { dual segmentation }\end{array}$ \\
\hline
\end{tabular}

\subsection{Object detection}

Object detection is the method for recognizing the non stationary or moving object in a video sequence [4] [5] [6].

\subsubsection{Optical flow}

The area of the image is computed and clustering of those fields are done according to the presence of the object in image. Optical flow process calculates the movement of objects among 2 image sequence which is occupied at times $t$ and $t+d t$ at every single location. This method helps in detecting the moving object with respect to the background, but not suitable for large quantity of calculation and sensitive to noise [4].

\subsubsection{Background subtraction}

Background subtraction is the most commonly used process for detecting moving objects. In this process background and foreground objects are separated to detect the non-stationary objects [7]. 

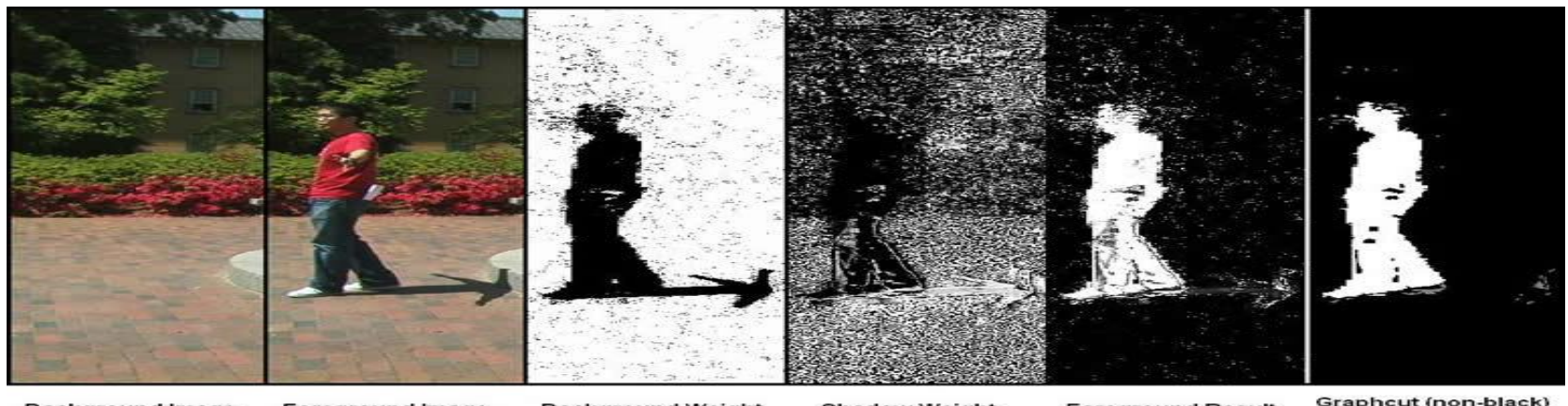

Background Weight

Shadow weight

Foreground Result

Graphcut (non-black)
Blob finding (white)

Figure 4. Background subtraction techniques

\subsubsection{Frame difference}

Detection of moving object from a sequence of frames captured from a static camera is widely performed by frame difference method. The objective of the approach is to detect the moving objects from the difference between the existing frame and the reference frame

$f(a, b)=\left\{\begin{array}{l}1 \ldots \text { ifIn }(a, b)-I_{n+1}(a, b)>T \\ \text { O...Otherwise }\end{array}\right.$

\subsubsection{Double difference}

It is also known as temporal difference. In double difference current frame pixel is subtract with its prior frame pixel or immediate next frame pixel. If the difference value is larger than the threshold value than it is reproduced as the foreground pixel and remaining pixel values will be considered as the background.

Table-2 Comparative study of object detection technique

\begin{tabular}{|l|l|l|}
\hline Object detection technique & Advantages & Limitations \\
\hline Optical flow & $\begin{array}{l}\text { Broad information regarding the } \\
\text { movement of the object }\end{array}$ & $\begin{array}{l}\text { Huge calculation and not good for } \\
\text { real time occlusion handling. Require } \\
\text { large amount of calculation. }\end{array}$ \\
\hline Background Subtraction & $\begin{array}{l}\text { It can reach real time processing. Low } \\
\text { memory requirement }\end{array}$ & $\begin{array}{l}\text { It does not survive with multimodal } \\
\text { Background. } \\
\text { Very sensitive to the change s in the } \\
\text { external environment and has poor } \\
\text { anti-interference ability. }\end{array}$ \\
\hline Frame Difference & $\begin{array}{l}\text { Perform well for static background. } \\
\text { High accuracy. Easiest method. }\end{array}$ & $\begin{array}{l}\text { It must require background without } \\
\text { moving objects. Method having } \\
\text { Computational time low to moderate }\end{array}$ \\
\hline
\end{tabular}

\subsection{Object tracking}

Some of the constraints that generally imposed during object tracking are object motion is smooth with no abrupt changes, no sudden changes in the background, gradual changes in the appearance of object, fixed camera, number and size of objects, limited amount of occlusion. For avoiding these issues, the task is to find an appropriate visual description that makes the object distinguished from other objects and from the background. The second issue is the appearance and shape can also be changed due to perspective effect that means objects farther from the camera appears smaller than those near to the camera. Shadows and reflections are also one of the difficulties to handle because motion, shape and background are more sensitive for a shadow on the ground which behaves and appears like the object that casts it [9]. The features of a tracking algorithm is to detect all the objects that enter or moved in the scene and also capable to differentiate between multiple objects that are present in the scene at the same time. For this the unique label assigned to each object must be maintained.

\subsubsection{Point tracking approach}

Moving objects are usually denoted by their feature point in the image sequence during tracking. Point tracking does not suit for occlusion condition and false object detection. It is capable of dealing with very small objects. Some approaches based on point tracking are kalman filter, particle filter, multiple hypothesis tracking [10]. In kalman filter, it consist of two phases, one is prediction and another one is correction i.e. prediction of the next state using the current set of observations then change the 
current set of predicted measurements [11]. Particle filter uses contours, color features, texture mapping. It generates all the models for one variable before moving to the next variable and take advantage when variables are generated dynamically. Multiple hypothesis tracking is an iterative algorithm begins with a set of existing track hypothesis. It is capable of tracking multiple objects, ability to tracks for objects enter and exit of field of view and also handles occlusions. [12].

\subsubsection{Kernel based tracking approach}

Kernel based tracking is typically performed to determine objects in motion, which is symbolized by elementary object area, since frame to frame [13-14]. There are different methods for kernel tracking like simple template matching, mean shift method, support vector machine, layering based tracking. In simple template matching, reference image is matched with the frame that is separated from the video and can track only single object. Mean shift method is based on region of interest, which is defined by the rectangular window in an initial frame. SVM have positive samples and negative samples. Positive samples contain tracked object and negative samples contain all the remaining images that are not tracked.

\subsubsection{Shape based tracking}

Certain objects would have composite shapes like hand, finger, shoulder, nose etc. which cannot be demonstrated by exact geometric shapes. The goal of this technique is to identify the moving object state in each and all frame through object model produced in the prior frame [15]. This model is capable of handling large variety of object, shapes, also deals with object split and merge. It is divided into two categories i.e. contour tracking and shape matching. Shape matching is similar to the template based tracking in kernel approach. Models object are in the form of density functions, object edges. Contour tracking is in the form of state space models i.e. the parameters of shape and the motion of the contour. Contour defines the boundary of an object. The region which comes inside the contour is known as the silhouette of the object. It is suitable for tracking complex non rigid shapes [11].

\subsubsection{Feature based tracking}

Feature based tracking is the most widely used tracking technique now a days. It is mainly divided into 2 steps. First phase is to extract the features of the entity like centroid, shape, color etc. Second step is to match those features in every frame .

Table-3 Comparative study of object tracking techniques

\begin{tabular}{|c|c|c|c|}
\hline Tracking techniques & Method Description & Advantage & Limitation \\
\hline \multirow[t]{2}{*}{ Point tracking } & Kalman filter & $\begin{array}{l}\text { Track points in noisy } \\
\text { image } \\
\text { Balance between } \\
\text { predicted values and } \\
\text { noisy measurements. }\end{array}$ & $\begin{array}{l}\text { State variables is distributed } \\
\text { by Gaussian }\end{array}$ \\
\hline & Particle filter & $\begin{array}{l}\text { Solve the problem of } \\
\text { kalman filter }\end{array}$ & $\begin{array}{l}\text { State of single image is } \\
\text { estimated }\end{array}$ \\
\hline \multirow[t]{2}{*}{ Kernel tracking } & Color histogram & $\begin{array}{l}\text { Robust to occlusion, } \\
\text { distraction. }\end{array}$ & $\begin{array}{l}\text { Don't give } \text { good } \\
\text { performance when object } \\
\text { and its background have } \\
\text { similar color }\end{array}$ \\
\hline & Mean Shift Method & $\begin{array}{l}\text { Accuracy of target } \\
\text { representation and } \\
\text { localization } \\
\text { Object motion by } \\
\text { translation and scaling }\end{array}$ & $\begin{array}{l}\text { Tracking only single object. } \\
\text { Necessity of a physical } \\
\text { initialization. }\end{array}$ \\
\hline \multirow[t]{2}{*}{ Shape tracking } & Contour tracking & $\begin{array}{l}\text { Object shape is } \\
\text { implicitly modeled }\end{array}$ & $\begin{array}{l}\text { Fail to show some of the } \\
\text { heights [16] }\end{array}$ \\
\hline & Shape matching & $\begin{array}{l}\text { Less sensitive to } \\
\text { appearance }\end{array}$ & Training is required \\
\hline Feature based & Color feature & $\begin{array}{l}\text { Frames are divided } \\
\text { into regions according } \\
\text { to color }\end{array}$ & $\begin{array}{l}\text { Can't identify when object } \\
\text { have same color }\end{array}$ \\
\hline
\end{tabular}




\section{CONCLUSION}

In this paper methods are described with merit and demerit, some methods are useful which decrease calculation time and increase efficiency. Tracking is a process locating an object in successive frames over time and it is useful in many applications such as security surveillance, video communication and traffic control also finding suspicious object. For illustration, the point trackers involve detection in every frame while kernel based tracking or contours based tracking require detection only when the object first appears in the scene. Advance study may open the door to find efficient algorithms to reduce computational cost and to decrease the time required for detecting the object for variety of videos containing diversified characteristics and increase accuracy rate.

\section{REFERENCES}

[1] W. X. Kang, Q. Q Yang, R. R Liang, "The Comparative Research on Image Segmentation Algorithm”, IEEE Conference on ETCS, $2009,703-707$.

[2] N. R. Pal, S. K. Pal, “A Review on Image Segmentation Techniques, Pattern Recognition”, 26(9), 1993, 1277-1294.

[3] K. K. Singh, A. Singh, “A Study of Image Segmentation Algorithms for Different Types of Image”, International Journal of Computer Science, 7(5), 2010.

[4] G. Sindhuja, R. Devi, "A Survey on Detection and Tracking of Objects in Video Sequence", International Journal of Engineering Research and General Science, 3(2), 2015.

[5] K. A. Joshi, D. G. Thakore, "A Survey on Moving Object Detection and Tracking in Video Surveillance System”, International Journal of Soft Computing and Engineering, 2(3), 2012

[6] V. Arunachalam, I. Sorimuthu, V. Raja Gopal and B. Sankaragomathi, "Automatic Fast Video Object Detection And Tracking On Video Surveillance System”, ICTACT Journal On Image And Video Processing, 3(1), 2012, ISSN: 0976-9102.

[7] Sen-Ching S. Cheung and Chandrika Kamath, "Robust techniques for background subtraction in urban traffic video".

[8] I. Pathan, C. Chauhan, "A Survey on Moving Object Detection and Tracking Methods", International Journal of Computer Science and Information Technologies, 6(6), 2015, 5212-5215.

[9] A. S. Jalal, V. Singh, "The State of-the-Art in Visual Object Tracking, International journal of computing and informatics”, 36(3), 2012, 227-248.

[10] J. J. Athanesious, P. Suresh, "Systematic Survey on Object Tracking Methods in Video", International Journal of Advanced Research in Computer Engineering \& Technology (IJARCET),2012, 242-247.

[11] A. Yilmaz, O. Javed, M. Shah, "Object Tracking: A Survey, ACM Computing Survey”, 38(4), 2006, 1-45.

[12] H. A. Patel, D. G. Thakore, "Moving Object Tracking Using Kalman Filter", International Journal of Computer Science and Mobile Computing , $2013,326-332$.

[13] J. J. Athanesious, Suresh P, "Implementation and Comparison of Kernel and Silhouette Based Object Tracking”, International Journal of Advanced Research in Computer Engineering \& Technology, 2013, 1298-1303.

[14] Sunitha M.R, H.N Jayanna, Ramegowda, "Human path tracing using combined Color and Centroid feature in a Video", International Journal of Applied Engineering Research, 12(9), 2017, 1848-1854.

[15] P Dokladal, R Enficiaud, E. Dejnozkova , "Contour based object tracking with gradient-based contour attraction field", IEEE International Conference on Acoustics, Speech, and Signal Processing ,2004, 17-20.

[16] X. Lu, Li Song, Songyu Yu, Nam Ling, "Object Contour Tracking Using Multi-feature Fusion based Particle Filter", IEEE Conference on Industrial Electronics and Applications, 2012, $237-242$. 